\title{
Numerical Investigation of Heat Transfer of CuO Nanofluid Using Eulerian-Eulerian Two Phase Model
}

\author{
Farhad Abbassi Amiri, Mohsen Nazari, Mohammad Mohsen Shahmardan \\ Mechanical Engineering, Shahrood University, Shahrood, Iran \\ Email address: \\ Farhad_a.abbassi@yahoo.com (F. A. Amiri)
}

\section{To cite this article:}

Farhad Abbassi Amiri, Mohsen Nazari, Mohammad Mohsen Shahmardan. Numerical Investigation of Heat Transfer of CuO Nanofluid Using Eulerian-Eulerian Two Phase Model. International Journal of Mechanical Engineering and Applications. Vol. 5, No. 5, 2017, pp. $259-268$. doi: 10.11648/j.ijmea.20170505.14

Received: September 8, 2017; Accepted: September 20, 2017; Published: September 26, 2017

\begin{abstract}
In this study, laminar forced convection of $\mathrm{CuO}$ nanofluid is numerically investigated in sudden expansion microchannel with expansion ratio of 3:1 and isotherm walls. The importance and developments of microfluidic devices, like expansion microchannel, has caused that the investigation of the flow and the heat transfer of nanofluid in sudden expansion microchannel to be so important. On the other hand, the two phase models can be used instead of single phase model very well. Among two phase models, Eulerian-Eulerian model is very efficient because of considering the relative velocity and temperature of the phases and the nanoparticle concentration distribution. An Eulerian two-fluid model is considered to simulate the nanofluid flow inside the microchannel and the governing mass, momentum and energy equations for both phases are solved using the finite volume method. It can be observed that the Eulerian two phase model of the $\mathrm{CuO}$ nanofluid enhances the heat transfer instead of using pure water as a coolant. Reynolds number and nanoparticle volume concentration increase the average Nusselt number, while the pressure drop increases only slightly. Also, the heat transfer increases with decrease in the nanoparticle diameter.
\end{abstract}

Keywords: Nanofluid, Heat Transfer, Microchannel, Two Phase, Eulerian-Eulerian

\section{Introduction}

Choi is the first researcher that has used the nanofluid [1]. Concentration on the modeling of the thermal conductivity of nanofluid has thrived by the other researchers [2-4]. Recently, the concentration is on the heat transfer and fluid flow behavior of nanofluid.

There are many experimental works for nanofluid on macro and micro-scales [5-8]. Wen and Ding [5] studied the heat transfer of $\mathrm{Al}_{2} \mathrm{O}_{3}$-water nanofluid in a copper tube under the constant heat flux. Their measurements in the heat transfer of $\mathrm{Al}_{2} \mathrm{O}_{3}$-water nanofluid showed an enhancement in the entrance region of the tube. They explained that the particle migration reduces the thickness of the thermal boundary layer and causes this behavior in the heat transfer of the nanofluid. Heris et al. [6] investigated $\mathrm{CuO}$-water and $\mathrm{Al}_{2} \mathrm{O}_{3}$-water nanofluids in an annular tube. They compared the results of the experimental study and the homogeneous model and realized the under-estimation of the homogeneous model in the heat transfer enhancement, especially in higher nanoparticle volume concentration. Homogeneous (single phase) and two-phase models are commonly used in the numerical study of the heat transfer and fluid flow of the nanofluid. In single phase model, the velocity and temperature of the base fluid and the particles are the same. Most studies in this field are done by using the single phase model $[3,9,10]$. In two phase model, the base fluid and particles are considered as two different phases in despite of the single phase model and both phases have different velocities and temperatures. Also, the interactions between the phases are so important in the governing equations of the two phase models.

Behzadmehr et al. [11] studied the turbulent convection of the nanofluid in a circular tube by using a two phase mixture model. After comparing their results with an experimental study, they reported that the mixture model has more coincidence to the experimental study in compared to the homogeneous model. Mirmasoumi and Behzadmehr [12] also used the two phase mixture model for studying the mixed convection of the nanofluid in a tube. Mirmasoumi 
and Behzadmehr [13] and Akbarinia and Laur [14] studied the effect of the nanoparticle size on the mixed convection of the nanofluid by the mixture model. An increase in the heat transfer of the nanofluid with decrease in the nanoparticle size was observed in both studies. Kurowski et al. [15] simulated the nanofluid flow by three different mixture, homogeneous and Eulerian-Lagrangian models in a minichannel. The results of all models were almost the same. Fard et al. [16] used single and two phase models for studying the heat transfer of the nanofluid inside a tube. They investigated a $0.2 \% \mathrm{CuO}$-water and compared the results with experimental ones and reported that the average relative error between the two phase model and experimental data was $8 \%$ while it was $16 \%$ for the single phase model.

Keshavarz and Mohammadi [17] investigated the thermal performance of $\mathrm{Al}_{2} \mathrm{O}_{3}$-water nanofluid in minichannel heat sink by using single and two phase models. In their study, it has been observed that the two-phase models have more coincidence with the experimental results in comparison to the single phase model, however, it is not sensible in low volume concentration but by increasing the particle volume concentration up to $1 \%$ or in high Reynolds number, the deviation between the single phase model and the experimental data increases. Shariat et al. [18] studied the impact of nanoparticle mean diameter and the buoyancy force on laminar mixed convection nanofluid flow in an elliptic duct by using two-phase mixture model. They reported that in certain Reynolds and Richardson numbers, the average Nusselt number increases with decrease in the nanoparticle diameter as well as with increase in Richardson number. A. Ramiar et al. [19] optimized the heat transfer of two phase modeling of nanofluid in a sinusoidal wavy channel using Artificial Bee Colony technique. A two phase model is applied to investigate different governing parameters, namely: Reynolds number, nonofluids volume fraction and amplitude of the wavy wall. For optimization process, a recent spot-lighted method, called Artificial Bee Colony (ABC) algorithm, is applied, and the results are shown to be in a good accuracy in comparison with another well-known heuristic method, i.e. particle swarm optimization (PSO). The results have indicated that the effect of utilizing nanoparticles and increasing Reynolds number is more intensified on growing the average Nusselt number than variations of the amplitude of the wavy wall. Omid Ali Akbari et al. [20] used a modified two-phase mixture model of nanofluid flow and heat transfer in a 3-D curved microtube. This study numerically investigated the laminar mixed-convection heat transfer of different water-copper nanofluids inside a microtube with curvature angle of $90^{\circ}$, using a finite volume method. The results showed that the heat transfer and hydraulics behavior of nanofluids in curved geometries is to some extent different with other geometries and flat surfaces due to presence of buoyancy and centrifugal forces at the same time.

P. Hanafizade et al. [21] studied the single and two-phase models for magnetite nanofluid forced convection in a tube. This study has compared two-phase and single-phase approaches in simulating forced convective heat transfer of $\mathrm{Fe}_{3} \mathrm{O}_{4}$-water nanofluid in both developing and fully developed regions of a tube under constant heat flux. Results of the two-phase models have been compared with that of the best single-phase model and the collected experimental data. It is concluded that considering the Brownian motion effect in the static Maxwell model significantly improves the accuracy. However, the presented correlations for thermal conductivity and viscosity lead to the closest results to the experimental data. M. Ziad Saghir et al. [22] investigated Two-phase and single phase models of flow of nanofluid in a square cavity. The numerical results have been compared with the specific condition of the experimental data, resulting in $1 \%$ discrepancy when using the single phase model. However, higher discrepancy with up to $10 \%$ when using the two phase model was found. Farhad Abbassi et al.[23] have studied the flow Bifurcation of the $\mathrm{CuO}$ nanofluid in the microchannel and reported the average Nusselt number enhancement with decrease in the expansion ratio of the microchannel.

In this work, the laminar forced convection of the $\mathrm{CuO}$ nanofluid in a sudden expansion microchannel with expansion ratio of 3:1 and isotherm walls is studied by twophase model of the Eulerian-Eulerian. The governing equations of mass, momentum and energy are discretized using the finite volume method and the modified SIMPLE algorithm is used for solving the flow equations of both phases. The modified SIMPLE algorithm has been created as a new strategy in order to solve the governing equations and accelerates the SIMPLE algorithm. The effects of the nanoparticle size and volume concentration and Reynolds number on the average Nusselt number are investigated. The Sudden expansion microchannels can be used as a microfluidic rectifier, like: Tesla rectifier, simple nozzle/diffuser structures and cascaded nozzle/diffuser structures. Because of the considering the relative velocity and temerature between phases and the nanoparticle volume concentration distribution, Eulerian-Eulerian model is more efficient in comparison to the other two phase model.

Numerical investigation of nanofluid flow and heat transfer in a sudden expansion microchannel by using the Eulerian-Eulerian two phase model and applying the modified SIMPLE algorithm is a new work in the field of nanofluid heat transfer in a microfluidic system.

\section{Governing Equations}

The geometry of this study is a sudden expansion microchannel with expansion ratio of $3: 1$ that is shown in Figure 1. The laminar flow of considered nanofluid that is the mixture of water and copper nanoparticles, enters the microchannel with uniform velocity and temperature and exchanges heat with the isothermal walls of the microchannel. The upstream length and height of the microchannel $\left(L_{1}, h\right)$, the downstream length and height of microchannel $\left(L_{2}, h\right)$ and the reattachment length after the expansion region $\left(L_{r}\right)$ are shown in Figure 1. 
L2

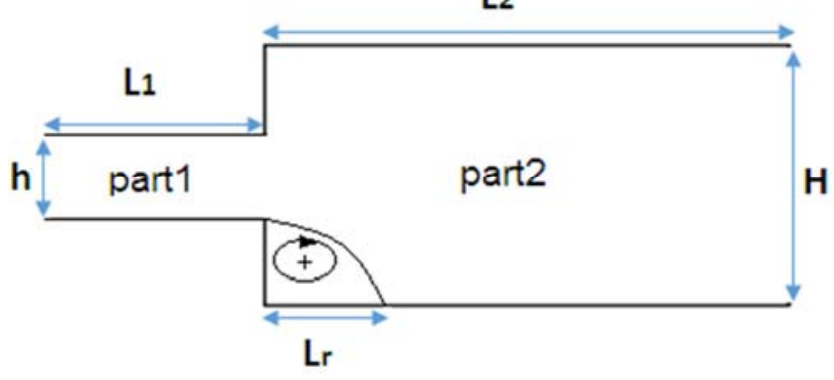

Figure 1. The sudden expansion microchannel.

Considering the steady state, laminar and EulerianEulerian two phase model, the governing equations for the base liquid and nanoparticle phases can be written as follows.

\subsection{Continuity Equations}

Continuity equations for solid and liquid phases in the cartesian coordinate system are as follows:

$$
\begin{gathered}
\frac{\partial\left(\varphi_{l} \rho_{l} u_{l} u_{l}\right)}{\partial x}+\frac{\partial\left(\varphi_{l} \rho_{l} v_{l} u_{l}\right)}{\partial y}=-\varphi_{l} \frac{\partial p}{\partial x}+\frac{\partial}{\partial x}\left(\varphi_{l} \mu_{l} \frac{\partial u_{l}}{\partial x}\right)+\frac{\partial}{\partial y}\left(\varphi_{l} \mu_{l} \frac{\partial u_{l}}{\partial y}\right)+\left(F_{d}\right)_{x}+\left(F_{v m}\right)_{x} \\
\frac{\partial\left(\varphi_{p} \rho_{p} u_{p} u_{p}\right)}{\partial x}+\frac{\partial\left(\varphi_{p} \rho_{p} v_{p} u_{p}\right)}{\partial y}=-\varphi_{p} \frac{\partial p}{\partial x}+\frac{\partial}{\partial x}\left(\varphi_{p} \mu_{p} \frac{\partial u_{p}}{\partial x}\right)+\frac{\partial}{\partial y}\left(\varphi_{p} \mu_{p} \frac{\partial u_{p}}{\partial y}\right)+\left(F_{c o l}\right)_{x}-\left(F_{d}\right)_{x}-\left(F_{v m}\right)_{x}
\end{gathered}
$$

Where $\left(F_{c o l}\right)_{x},\left(F_{d}\right)_{x},\left(F_{v m}\right)_{x}, \mathrm{P}$ and $\mu$ are the particleparticle interaction, drag, virtual mass (added mass) forces, pressure and viscosity, respectively. Here, the lift force between the phases can be neglected because of the small size of the nanoparticles.
Equation in the Y-direction

In these equations, the gravitational force is neglected because of the small size of the microchannel and the equations can be written as follows

$$
\begin{gathered}
\frac{\partial\left(\varphi_{l} \rho_{l} u_{l} v_{l}\right)}{\partial x}+\frac{\partial\left(\varphi_{l} \rho_{l} v_{l} v_{l}\right)}{\partial y}=-\varphi_{l} \frac{\partial p}{\partial y}+\frac{\partial}{\partial x}\left(\varphi_{l} \mu_{l} \frac{\partial v_{l}}{\partial x}\right)+\frac{\partial}{\partial y}\left(\varphi_{l} \mu_{l} \frac{\partial v_{l}}{\partial y}\right)+\left(F_{d}\right)_{y}+\left(F_{v m}\right)_{y} \\
\frac{\partial\left(\varphi_{p} \rho_{p} u_{p} v_{p}\right)}{\partial x}+\frac{\partial\left(\varphi_{p} \rho_{p} v_{p} v_{p}\right)}{\partial y}=-\varphi_{p} \frac{\partial p}{\partial y}+\frac{\partial}{\partial x}\left(\varphi_{p} \mu_{p} \frac{\partial v_{p}}{\partial x}\right)+\frac{\partial}{\partial y}\left(\varphi_{p} \mu_{p} \frac{\partial v_{p}}{\partial y}\right)+\left(F_{c o l}\right)_{y}-\left(F_{d}\right)_{y}-\left(F_{v m}\right)_{y}
\end{gathered}
$$

The force terms in the momentum equations are defined as

$$
\begin{gathered}
F_{d}=-\beta\left(\vec{V}_{l}-\vec{V}_{p}\right) \\
F_{v m}=0.5 \varphi_{p} \rho_{l} \frac{D}{D t}\left(\vec{V}_{l}-\vec{V}_{p}\right) \\
F_{c o l}=G\left(\varphi_{l}\right) \vec{\nabla} \varphi_{l}
\end{gathered}
$$

Where $\vec{V}$ is the velocity vector. particle interaction modulus and the nanoparticle diameter, respectively.

\subsection{Energy Equations}

The base fluid and particle phases are considered as incompressible fluids and the viscous dissipation and radiation are neglected. So, the energy equations in cartesian coordinate system can be written as

Here, $\beta, \mathrm{G}$ and $d_{p}$ are the friction coefficient, the particle-

$$
\begin{aligned}
\frac{\partial}{\partial x}\left(\varphi_{l} \rho_{l} u_{l} c_{p l} T_{l}\right)+\frac{\partial}{\partial y}\left(\varphi_{l} \rho_{l} v_{l} c_{p l} T_{l}\right) & =\frac{\partial}{\partial x}\left(\varphi_{l} k_{e f f, l} \frac{\partial T_{l}}{\partial x}\right)+\frac{\partial}{\partial y}\left(\varphi_{l} k_{e f f, l} \frac{\partial T_{l}}{\partial y}\right)-h_{v}\left(T_{l}-T_{p}\right) \\
\frac{\partial}{\partial x}\left(\varphi_{p} \rho_{p} u_{p} c_{p_{p}} T_{p}\right)+\frac{\partial}{\partial y}\left(\varphi_{p} \rho_{p} v_{p} c_{p_{p}} T_{p}\right) & =\frac{\partial}{\partial x}\left(\varphi_{p} k_{e f f, p} \frac{\partial T_{p}}{\partial x}\right)+\frac{\partial}{\partial y}\left(\varphi_{p} k_{e f f, p} \frac{\partial T_{p}}{\partial y}\right)+h_{v}\left(T_{l}-T_{p}\right)
\end{aligned}
$$

Here, T, $k_{e f f}, C_{p}$ and $h_{v}$ are the temperature, effective thermal conductivity, heat capacity at constant pressure and volume interphase heat transfer coefficient, respectively. For spherical nanoparticles $h_{v}$ can be calculated as

$$
h_{v}=\frac{6\left(1-\varphi_{l}\right)}{d_{p}} h_{p}
$$

Here, $h_{p}$ is the fluid-particle heat transfer coefficient that is obtained from experimental correlations.

The Nusselt number is calculated from the temperature difference between the nanofluid mean temperature and the microchannel walls:

$$
N u=\frac{\left(h D_{h}\right)}{k_{l}}=q^{\prime \prime} D_{h} / k_{l}\left(T_{w}-T_{m}\right)
$$

Here, $q^{\prime \prime}, \mathrm{h}$ and $D_{h}$ are the wall convective heat transfer 
flux, convective heat transfer coefficient and the microchannel hydraulic diameter, respectively. The mean temperature of the nanofluid is calculated as follow

$$
T_{m}=\frac{\sum_{i=1}^{p}\left(\int \rho_{i} u_{i} c_{p i} T_{i} d A\right)}{\sum_{i=1}^{p}\left(\int \rho_{i} u_{i} c_{p i} d A\right)}
$$

Where the integration is applied on the microchannel cross section.

According to the equations (11) and (12), the wall convective heat transfer flux can be obtained as

$$
q^{\prime \prime}=\left(\varphi_{l} k_{e f f, l} \frac{\partial T_{l}}{\partial y}\right)_{w}+\left(\varphi_{p} k_{e f f, p} \frac{\partial T_{p}}{\partial y}\right)_{w}
$$

Considering the local heat transfer coefficient, the average heat transfer coefficient is

$$
\bar{h}=\frac{1}{L} \int_{0}^{L} h d x
$$

\subsection{Non-Dimensionalization}

The non-dimensional form of the governing equations are obtained, using the following non-dimensional parameters:

$$
X=\frac{x}{D_{h}}, Y=\frac{y}{D_{h}}, U_{i}=\frac{u_{i}}{u_{\text {in }}}, V_{i}=\frac{v_{i}}{u_{\text {in }}}, P=\frac{p-p_{\text {in }}}{\rho_{l} u_{i n}{ }^{2}}, \theta_{i}=\frac{T_{i}-T_{i n}}{T_{w}-T_{i n}} \operatorname{Re}_{i}=\frac{u_{i n} D_{h} \rho_{i}}{\mu_{i}}, \operatorname{Pr}_{i}=\frac{\rho_{i} C_{p_{i}} v_{i}}{k_{i}}
$$

Where $\mathrm{i}=1, \mathrm{p}$ indicates the liquid and particle phases.

\subsection{Boundary Conditions}

Base fluid and the particle phases enter the microchannel with the same uniform axial velocity at the inlet. At the outlet of the microchannel, the velocity boundary condition is considered for the outflow of the both phases. In this work, the non-slip boundary condition at the walls is assumed for both phases.

For thermal boundary conditions, the microchannel walls are isothermal and the outflow temperature boundary condition is considered for both phases. Since the length of the microchannel is too long, the variations of the outflow velocity and temperature in $\mathrm{x}$-direction are assumed as follow

$$
\frac{\partial u}{\partial x}=0, \frac{\partial v}{\partial x}=0, \frac{\partial T}{\partial x}=0
$$

\section{Numerical Method}

In this study, the governing equations are solved numerically in Fortran. The finite volume method is used for discretizing the governing equations. The first order upwind scheme is used for discretizing the convection-diffusion term. The SIMPLE algorithm is used for the pressure-velocity coupling [24, 25] and the discretized governing equations are converted to a set of algebric equations that is solved iteratively. In this study, A new strategy is created in order to modify the SIMPLE algorithm by obtaining a new pressure correction equation with the combination of the contunity equations of base fluid and the particle phases. The underrelaxation coefficients are used for velocity and pressure in order to accelerate the convergence of the algorithm. In this algorithm, the source term of the pressure correction equation is considered as the convergence criterion.

\section{Grid Independence Study}

Independency of the results versus the number of grid points is investigated by calculating the average Nusselt number for various numbers of grid points and Reynolds numbers. Table 1 shows the average Nusselt numbers for four different numbers of grid points and $\mathrm{Re}=100$. The grid 3 is selected for this study as it can be seen that the average Nusselt numbers between the 3 and 4 grids have a negligible difference.

Table 1. Grid-independency study results for $\varphi_{p}=0.01$ and $R e=100$.

\begin{tabular}{lllll}
\hline \multirow{2}{*}{ Grid } & $\mathbf{1}$ & $\mathbf{2}$ & $\mathbf{3}$ & \multicolumn{1}{c}{$\mathbf{4}$} \\
\cline { 2 - 5 } & $\boldsymbol{N}_{\boldsymbol{y}} \times \boldsymbol{N}_{\boldsymbol{x}}$ & $\boldsymbol{N}_{\boldsymbol{y}} \times \boldsymbol{N}_{\boldsymbol{x}}$ & $\boldsymbol{N}_{\boldsymbol{y}} \times \boldsymbol{N}_{\boldsymbol{x}}$ & $\boldsymbol{N}_{\boldsymbol{y}} \times \boldsymbol{N}_{\boldsymbol{x}}$ \\
\hline Part1 & $10 \times 15$ & $20 \times 50$ & $30 \times 100$ & $40 \times 120$ \\
Part2 & $30 \times 45$ & $60 \times 150$ & $90 \times 300$ & $120 \times 360$ \\
All cells & 1500 & 10000 & 30000 & 48000 \\
Average Nusselt & 7.214875 & 7.654121 & 7.988713 & 8.014657 \\
\hline
\end{tabular}

\section{Validation}

To validate the results of this study, the reattachment length for the pure water $\left(\varphi_{p}=0.0\right)$ at different Reynolds numbers is calculated and compared to Scott et al. [26] results in order to investigate the accuracy of the code. Table 2 and Figure 2 show the reasonable coincidence between the different amounts of the reattachment length of these two studies and the maximum deviation is less than $4 \%$.
Table 2. Comparison between reattachment length of this study and Scott's one.

\begin{tabular}{lll}
\hline $\begin{array}{l}\text { Reynolds } \\
\text { number }(R e)\end{array}$ & Reattachment length $\frac{L_{r}}{h}$ & Reattachment length $\frac{L_{r}}{h}[26]$ \\
\hline 50 & 5.3 & 5.1 \\
100 & 9.3 & 9.7 \\
150 & 14.6 & 15 \\
200 & 20 & 20 \\
\hline
\end{tabular}




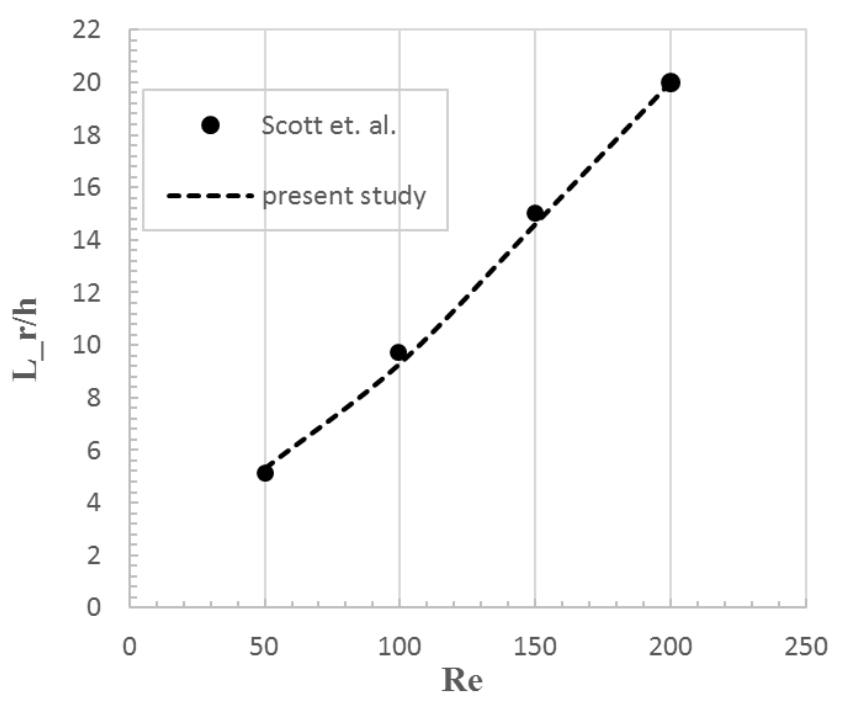

Figure 2. Comparison between reattachment length of this study and Scott's one for different Reynolds number.

\section{Results and Discussion}

\subsection{Distribution of Velocity, Temperature and Nanoparticle Volume Concentration}

Figures 3-5 show the distributions of velocity, temperature and nanoparticle volume concentration at $\varphi_{p}=0.01, \mathrm{Re}=100$ and $d_{p}=100 \mathrm{~nm}$ in the three main parts of sudden expansion microchannel including fully developed region of part 1 , fully developed region of part 2 and the sudden expansion section of the michrochannel.

\subsubsection{Velocity Distribution}

Fully developed Velocity profile

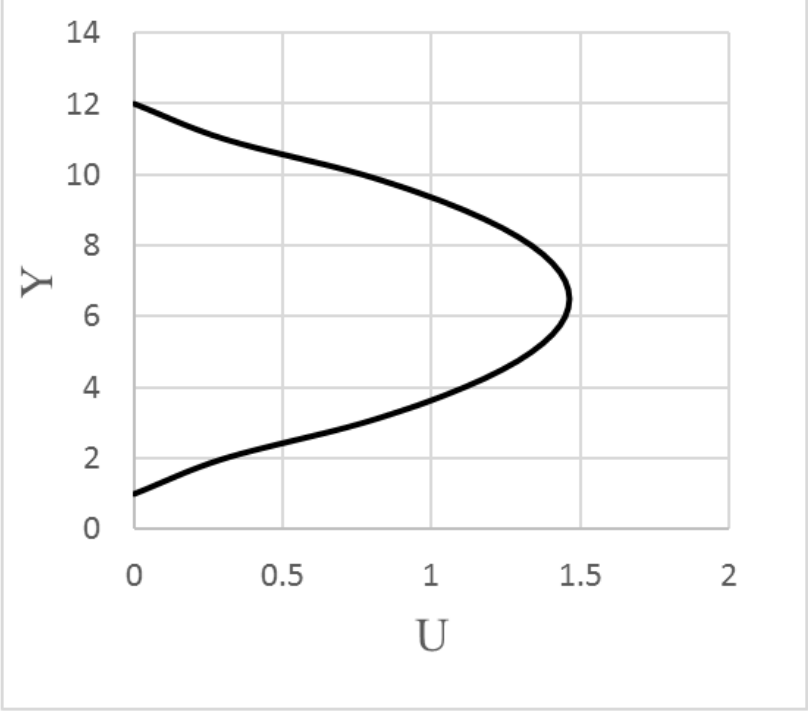

a) Fully developed region of part 1

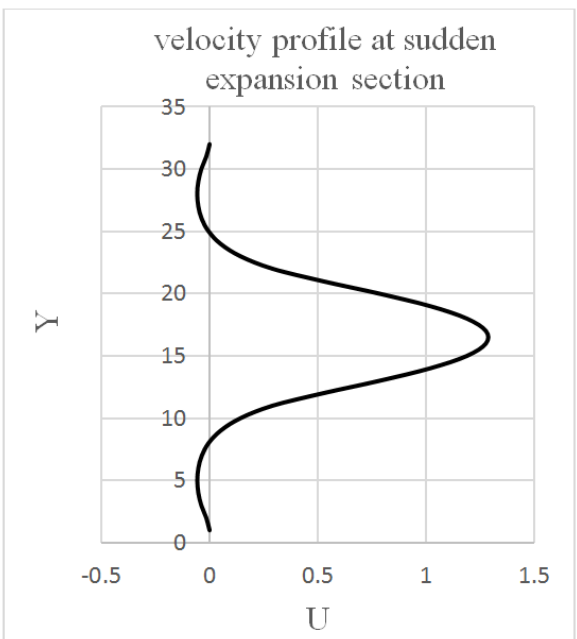

b) Sudden expansion section of microchannel

Fully developed velocity profile

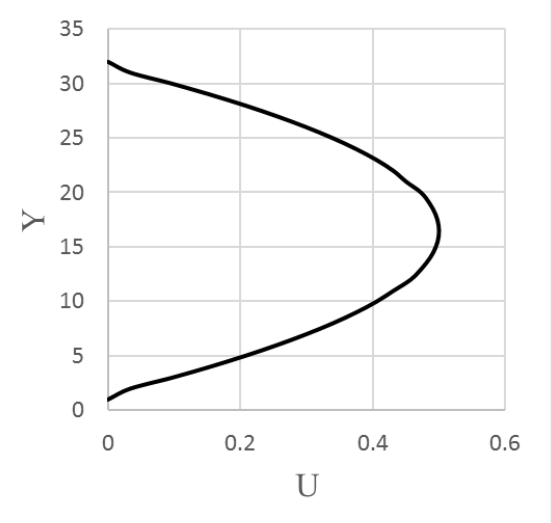

c) Fully developed region of part 2

Figure 3. Velocity distributions at three main regions of sudden expansion microchannel a) Fully developed region of part 1 b) Sudden expansion section of microchannel c) Fully developed region of part2.

\subsubsection{Temperature Distribution}

Fully developed temperature profile

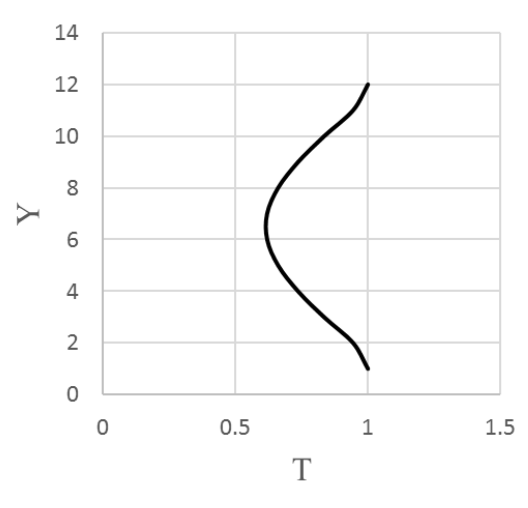

a) Fully developed region of part 1 


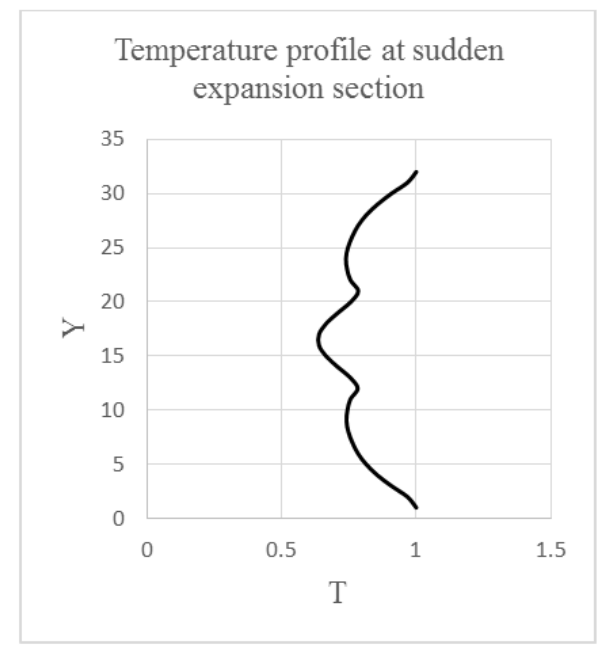

b) Sudden expansion section of microchannel

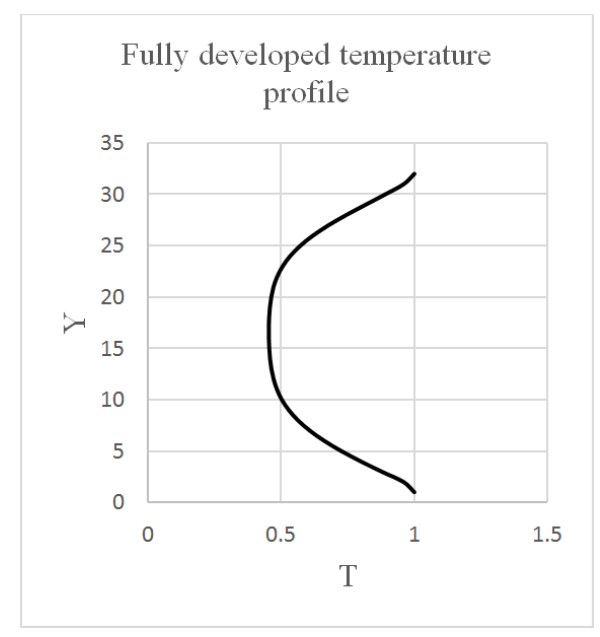

c) Fully developed region of part 2

Figure 4. Temperature distributions at three main regions of sudden expansion microchannel a) Fully developed region of part 1 b) Sudden expansion section of microchannel c) Fully developed region of part 2 .

\subsubsection{Distribution of Nanoparticle Volume Concentration}

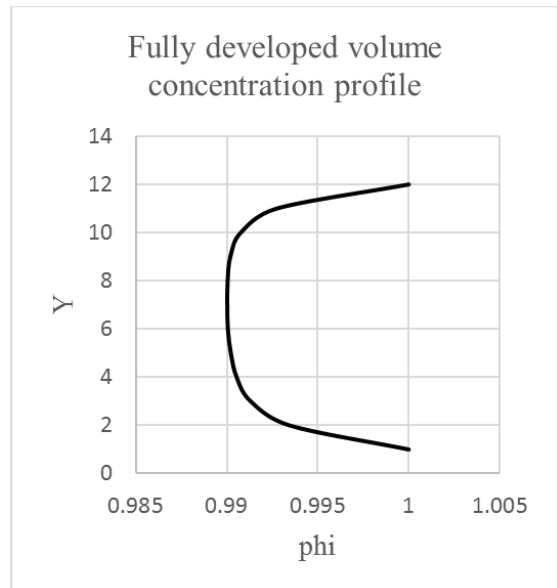

a) Fully developed region of part 1

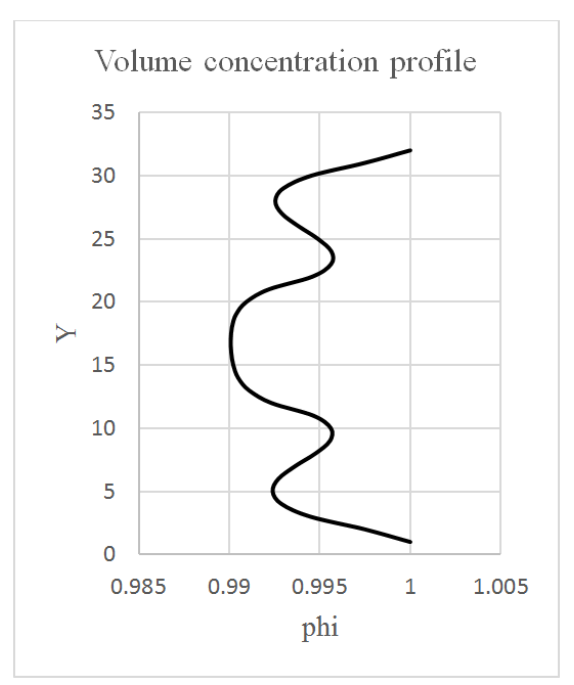

b) Sudden expansion section of microchannel

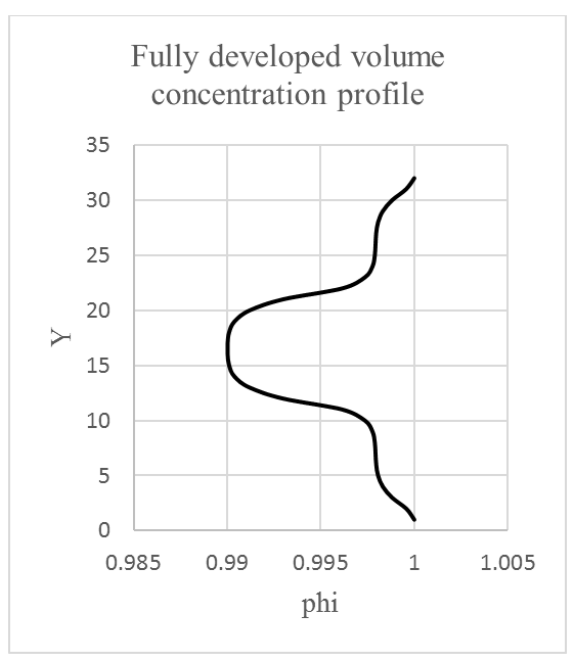

c) Fully developed region of part 2

Figure 5. Distribution of nanoparticle volume concentration at three main regions of sudden expansion microchannel a) Fully developed region of part 1 b) Sudden expansion section of microchannel c) Fully developed region of part 2 .

\subsection{Nanoparticles Effect on the Velocity and Temperature Field}

Figure 6 shows the nanoparticle effect on the velocity field at $\varphi_{p}=0.01$ and $\mathrm{Re}=50$ and the velocity contours of the nanofluid (base fluid and nanoparticle) and the pure water are compared to each other. It can be seen that nanoparticles have very small effect on the velocity distribution and incease the hydrodynamic entrance length slightly because of the very small size and volume concentration. Due to this reason, the nanofluid pressure drop increases slightly in comparison to the pure water. 
Base fluid phase

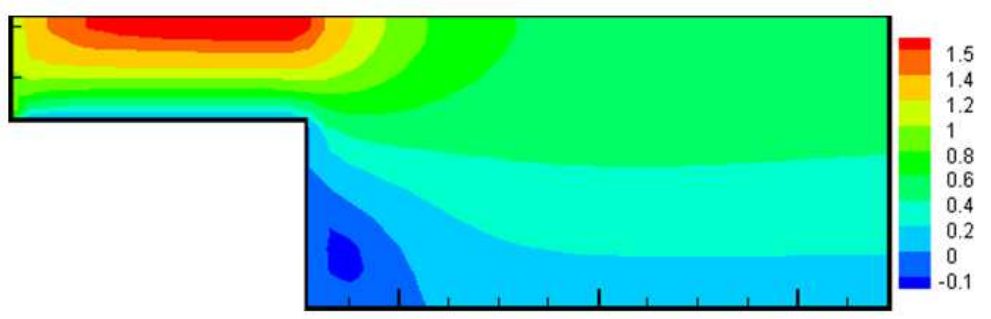

Nanoparticle phase

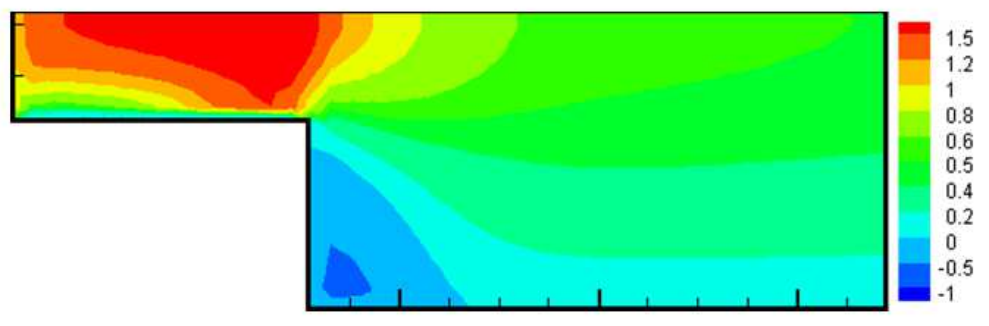

Pure water

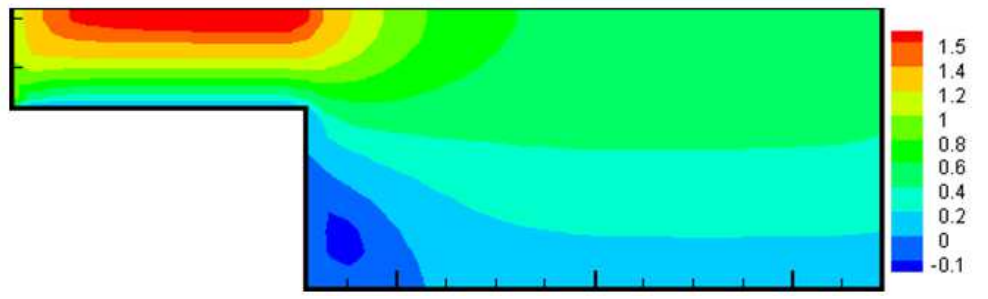

Figure 6. Velocity contours for nanofluid (base fluid and nanoparticle) and pure water at $\varphi_{p}=0.01$ and $R e=50$.

Base fluid phase

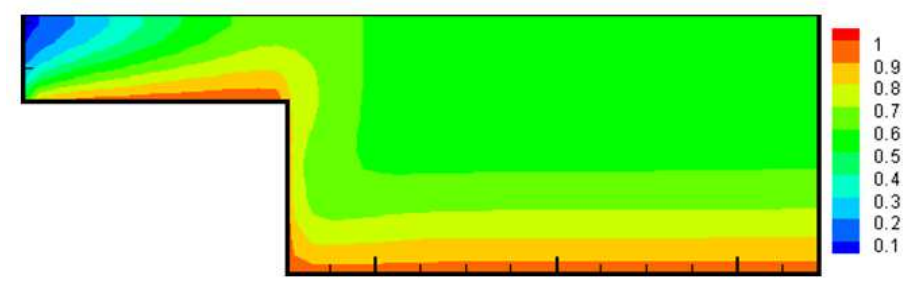

Nanoparticle phase

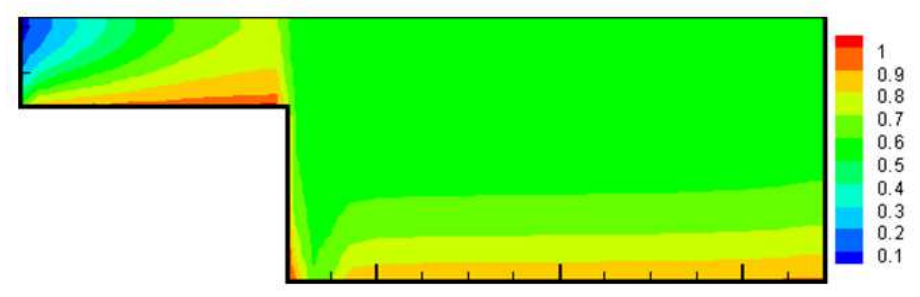

Pure water

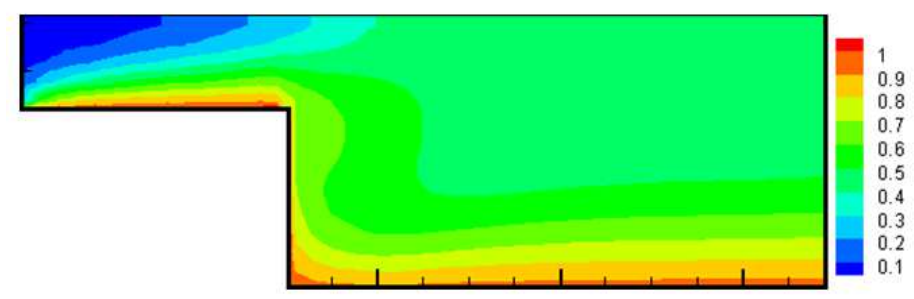

Figure 7. Temperature contours for nanofluid (base fluid and nanoparticle) and pure water at $\varphi_{p}=0.01$ and $R e=50$. 
Figure 7 shows the nanoparticle effect on the temperature field at $\varphi_{p}=0.01$ and $\mathrm{Re}=50$ and the temperature contours of the nanofluid (base fluid and nanoparticle) and the pure water are compared to each other. It has been observed that the development of the thermal boundary layer increases remarkably due to the presence of the nanoparticles that increase the fluid thermal conductivity and consequently increase the wall convective heat transfer flux. In this study, the distance that thermal boundary layer development of the nanofluid occurs, is $\frac{L_{2}}{6}$ after the sudden expansion section while this is $\frac{L_{2}}{4}$ for the pure water, so nanoparticles defer the thermal development.

\subsection{Nanoparticle Volume Concentration Effect on the Nanofluid Heat transfer}

Figure 8 illustrates the pressure drop for $d_{p}=100 \mathrm{~nm}$ when the nanoparticle volume concentration changes. From Figure 8 it has been observed that the pressure drop increases with increase of the particle volume concentration but it is small for all Reynolds numbers. The previous studies also reported this behavior of the pressure drop [7 and 8]. As it is said in the previous part, this behavior occurs due to the small effect of the nanoparticles on the velocity field.

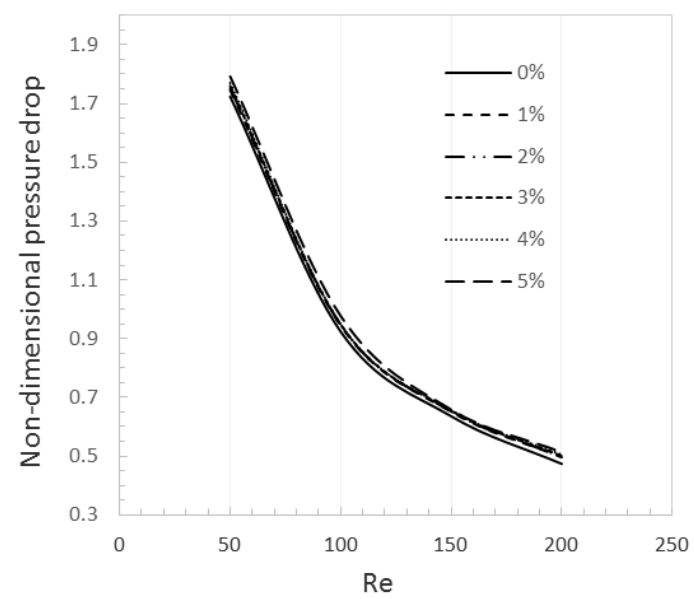

Figure 8. Non-dimensional pressure drop versus Reynolds number for different volume concentration and $d_{p}=100 \mathrm{~nm}$.

Figure 9 depicts the effect of Reynolds number and the nanoparticle volume concentration on the average Nusselt number for $d_{p}=100 \mathrm{~nm}$. It has been observed that the average Nusselt number increses with increase in Reynolds number and nanoparticle volume concentration. Therefore, this behavior of the nanofluid make it an effective working fluid in the cooling systems. Reynolds number increases the convection effects and the presence of more nanoparticles increases the thermal conductivity of the nanofluid and these reasons cause the higher heat transfer of it. Also, this result has been reported by the previous experimental and numerical studies for microchannels $[8,9]$.

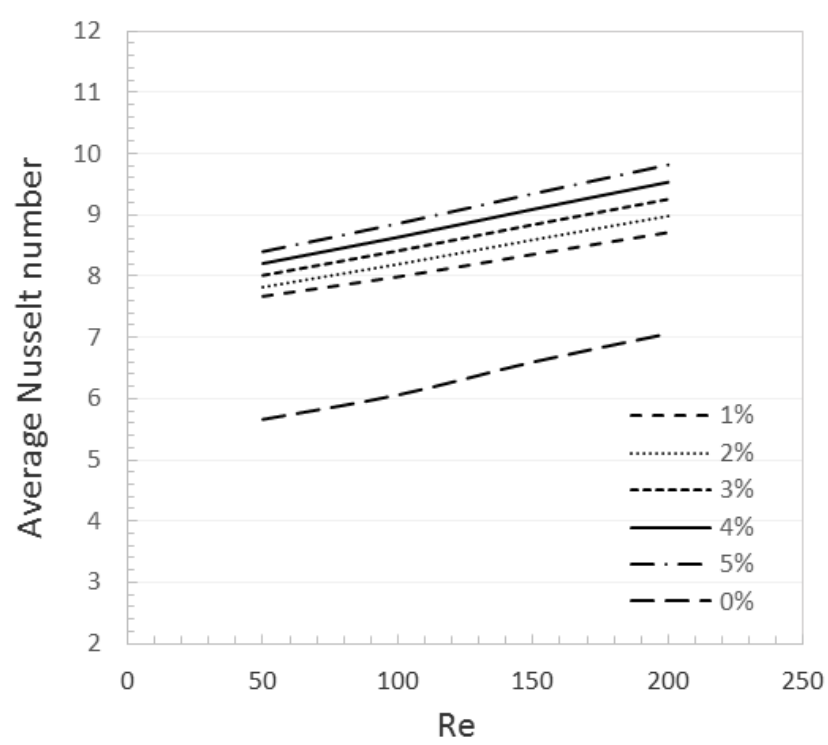

Figure 9. Average Nusselt number versus Reynolds number for different volume concentration and $d_{p}=100 \mathrm{~nm}$.

Figure 10 shows the effect of Reynolds number and nanoparticle volume concentration on the nanofluid average Nusselt number that has been normalized by the pure water Nusselt number for $d_{p}=100 \mathrm{~nm}$. As it is presented in the previous part, the presence of the nanoparticles increases the thermal conductivity of the nanofluid and consequently increases it's heat transfer. Also, this result can be seen from Figure 10 that the average Nusselt number ratio increases with an increase in the nanoparticle volume concentration for every Reynolds number. Another important observation in Figure 10 is that the lower Reynolds number affects more on increasing the heat transfer ratio for a definite nanoparticle volume concentration.

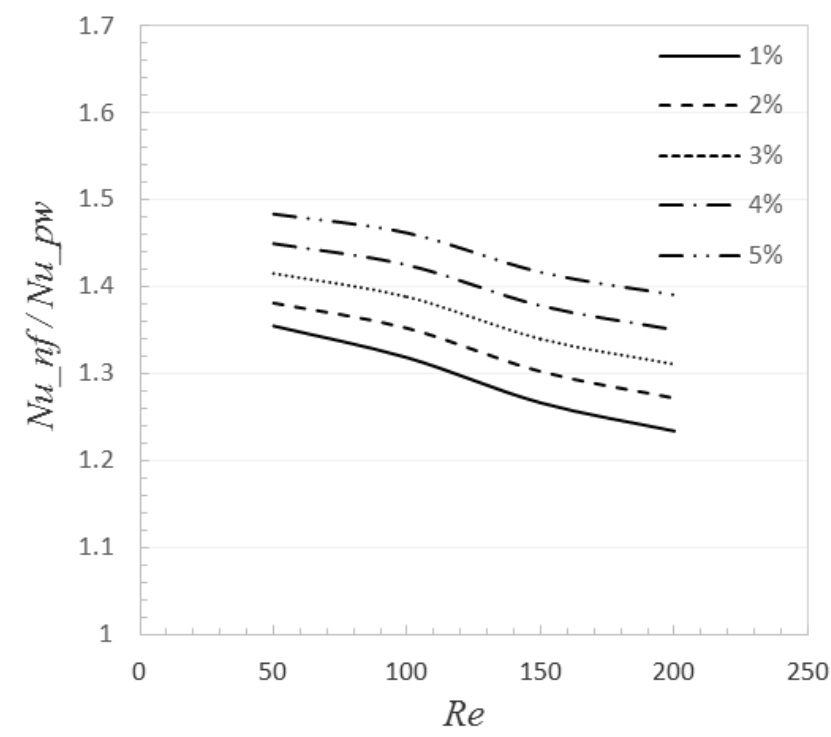

Figure 10. Nanofluid average Nusselt number normalized by the pure water Nusselt number versus Reynolds number for different nanoparticle volume concentration and $d_{p}=100 \mathrm{~nm}$. 


\subsection{Nanoparticle Diameter Effect on the Nanofluid Heat Transfer}

Figure 11 shows the nanoparticle diameter effect on the percentage enhancement in average Nusselt number in comparison to pure water flow for different nanoparticle volume concentration and $\mathrm{Re}=200$. In Figure 11 the enhancement of heat transfer increases with decrease in the nanoparticle diameter as well as with increase in the nanoparticle volume concentration. For instance, at $\varphi_{p}=0.02$, the copper nanoparticles with $d_{p}=50 \mathrm{~nm}$ increase the heat transfer enhancement $5 \%$ more than the copper nanoparticles with $d_{p}=100 \mathrm{~nm}$. In fact, By decreasing the nanoparticle size, the interaction face between nanoparticles and fluid increases that enhances the nanoparticle Brownian motion and causes the heat transfer enhancement.

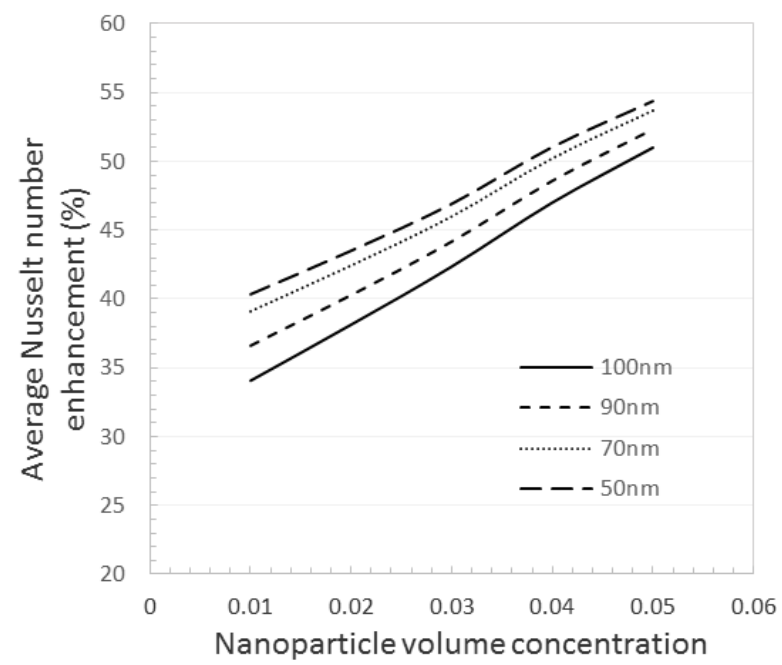

Figure 11. Percentage enhancement in average Nusselt number in comparison to pure water flow versus nanoparticle volume concentration for different nanoparticle diameter and $R e=200$.

\section{Conclusion}

In this study, laminar forced convection of $\mathrm{CuO}$ nanofluid is numerically investigated in sudden expansion microchannel with isotherm walls and expansion ratio of $3: 1$. A two-phase model is considered to simulate the nanofluid flow inside the microchannel. The finite volume method is used for solving the governing mass, momentum and energy equations of the both phases. In solving the equations for both phases, the SIMPLE algorithm is used and modified by combination of the continuity equations of the both phases and a new pressure correction equation is created in order to accelerate this algorithm. All in all, the Eulerian - Eulerian two-phase model of the $\mathrm{CuO}$ nanofluid shows higher heat transfer enhancement in comparison to pure water. The average nusselt number increases with increase in Reynolds number and nanoparticle volume concentration, while the increase in the pressure drop is small. For a constant volume concentration, the lower Reynolds number causes the larger average Nusselt number ratio. However, the average Nusselt number increases with decrease in the nanoparticle diameter so that a $2 \%$ copper-water nanofluid, for $50 \mathrm{~nm}$ particle size, the enhancement in heat transfer is 5\% more in comparison to 100 $\mathrm{nm}$ particle size. This investigation of $\mathrm{CuO}$ nanofluid heat transfer in sudden expansion microchannel is so important because of it's innovative numerical technique for solving the governing equations in the SIMPLE algorithm and using the Eulerian-Eulerian two phase model instead of single phase model very well.

\section{References}

[1] Choi SUS (1995) Enhancing thermal conductivity of fluids with nanoparticles. In: Siginer, Wang DA, HP (Eds.), Developments and Applications of Non-Newtonian Flows. ASME. (66): 99-105.

[2] Xuan Y, Li Q, Hu W (2003) Aggregation structure and thermal conductivity of nanofluids. AIChE J. (49): 10381043 .

[3] Koo J, Kleinstreuer C (2004) A new thermal conductivity model for nanofluids. J. Nanoparticles Res. (6): 577-588.

[4] Feng Y, Yu B, Xu P, Zou M (2007) The effective thermal conductivity of nanofluids based on the nanolayer and the aggregation of nanoparticles. J. Phys. D: Appl. Phys. (40): 3164-3171.

[5] Wen D, Ding Y (2004) Experimental investigation into convective heat transfer of nanofluids at the entrance region under laminar flow conditions. Int J Heat Mass Transfer. (47): 5181-5188.

[6] Heris SZ, Etemad SGh, Esfahany MN (2006) Experimental investigation of oxide nanofluids laminar flow convective heat transfer. J. International Communications in Heat and Mass Transfer. (33): 529-535.

[7] Jung JY, Oh HS, Kwak HY (2009) Forced convective heat transfer of nanofluids in microchannels. Int $\mathrm{J}$ Heat Mass Transfer. (52): 466-472.

[8] Wu X, Wu H, Cheng P (2009) Pressure drop and heat transfer of $\mathrm{Al}_{2} \mathrm{O}_{3}-\mathrm{H}_{2} \mathrm{O}$ nanofluids through silicon microchannels. $\mathrm{J}$. Micromech. Microeng. (19): 105-112.

[9] Li J, Kleinstreuer C (2008) Thermal performance of nanofluid flow in microchannels. Int J Heat Fluid Flow. (29): 12211232.

[10] Santra AK, Sen S, Chakraborty N (2009) Study of heat transfer due to laminar flow of copper-water nanofluid through two isothermally heated parallel plates. Int J Thermal Sci. (48): 391-400.

[11] Behzadmehr A, Saffar-Avval M, Galanis N (2007) Prediction of turbulent forced convection of a nanofluid in a tube with uniform heat flux using a two phase approach. Int J Heat Fluid Flow. (28): 211-219.

[12] Mirmasoumi S, Behzadmehr A (2008a) Numerical study of laminar mixed convection of a nanofluid in a horizontal tube using two-phase mixture model. J. Appl. Thermal Eng. (28): 717-727.

[13] Mirmasoumi S, Behzadmehr A (2008b) Effect of nanoparticles mean diameter on mixed convection heat transfer of a nanofluid in a horizontal tube. Int $\mathrm{J}$ Heat Fluid Flow. (29): 557-566. 
[14] Akbarinia A, Laur R (2009) Investigating the diameter of solid particles effects on a laminar nanofluid flow in a curved tube using a two phase approach. Int J Heat Fluid Flow. (30): 706-714.

[15] Kurowski L, Chmiel-Kurowska K, Thullie J (2009) Numerical simulation of heat transfer in nanofluids. J. Computer Aided Chemical Engineering. (26): 967-972.

[16] Fard MH, Esfahany MN, Talaie MR (2010) Numerical study of convective heat transfer of nanofluids in a circular tube two-phase model versus single-phase model. J. Int Commun. Heat Mass Transfer. (37): 91-97.

[17] Keshavarz M, Mohammadi R (2013) CFD modeling (comparing single and two-phase approaches) on thermal performance of $\mathrm{Al}_{2} \mathrm{O}_{3}$ /water nanofluid in mini-channel heat sink. J. International Communications in Heat and Mass Transfer. (25): 582-597.

[18] Shariat M, Mokhtari R, Akbarinia A, Rafee R, Sajjadi S. M (2014) Impact of nanoparticle mean diameter and the buoyancy force on laminar mixed convection nanofluid flow in an elliptic duct employing two phase mixture model. J. International Communications in Heat and Mass Transfer. (50): $15-24$

[19] A. Ramiar, P. Valinataj-Bahnemiri, S. A. Manavi (2015) Heat transfer optimization of two phase modeling of nanofluid in a sinusoidal wavy channel using Artificial Bee Colony technique. (18): 727-737.
[20] Omid Ali Akbar, Mohammad Reza safaei, Marjan Goodarzi (2016) A modified two-phase mixture model of nanofluid flow and heat transfer in a 3-D curved microtube. (27): 21752185 .

[21] P. Hanifzade, M. Ashjaee, M. Goharkhah, K. Montazeri (2017) The comparative study of single and two-phase models for magnetite nanofluid forced convection in a tube. (65): 5870 .

[22] M. Ziad Saghir, Amirhossein Ahadi, Tooraj Yousefi, Bahram Farahbakhsh (2016) Two-phase and single phase models of flow of nanofluid in a square cavity. (100): 372-380.

[23] Farhad A. Abbassi, Mohsen Nazari, M. M Shahmardan (2017) Numerical Study of Heat Transfer and Flow Bifurcation of $\mathrm{CuO}$ Nanofluid in Sudden Expansion Microchannel Using Two-Phase Model (7): 57-72.

[24] Patankar SV (1980) Numerical Heat Transfer and Fluid Flow. Hemisphere, Washington, DC.

[25] Versteeg HK, Malalasekera W (1995) An introduction to computational fluid dynamics the finite volume method. Longman Scientific and Technical, England.

[26] Scott PS, Mirza FA (1986) A Finite Element Analysis of Laminar Flows through Planar and Axisymmetric Abrupt Expansions. J. Computers \& Fluids. (14): 423-432. 